\title{
Generalized Route to Metal Nanoparticles with Liquid Behavior
}

Scott C. Warren, ${ }^{\dagger, \ddagger}$ Matthew J. Banholzer, ${ }^{\dagger}$ Liane S. Slaughter, ${ }^{\dagger}$ Emmanuel P.

Giannelis, ${ }^{\ddagger}$ Francis J. DiSalvo, ${ }^{\dagger}$ Ulrich B. Wiesner ${ }^{\ddagger, \star}$

${ }^{\dagger}$ Department of Chemistry \& Chemical Biology and ${ }^{\ddagger}$ Department of Materials Science \&

Engineering, Cornell University, Ithaca, NY 14853

\section{Supporting Information}

\section{Equipment}

Standard Schlenk line techniques were used for the synthesis of the ligand. All nanoparticles were synthesized under a nitrogen atmosphere. NMR spectra were acquired on a Varian Inova at $400 \mathrm{MHz}\left({ }^{1} \mathrm{H}\right)$ and $100 \mathrm{MHz}\left({ }^{13} \mathrm{C}\right)$. TGA was performed using a TA Instruments Q500 instrument equipped with an autosampler. Heating was performed at $10{ }^{\circ} \mathrm{C} / \mathrm{min}$. from $25{ }^{\circ} \mathrm{C}$ to $550{ }^{\circ} \mathrm{C}$ in an aluminum pan under flowing nitrogen. DSC was performed using a TA Instruments Q1000 instrument equipped with a LNCS and autosampler. Samples were tested in a heat $/ \mathrm{cool} /$ heat cycle ranging from $-150{ }^{\circ} \mathrm{C}$ to $130^{\circ} \mathrm{C}$ in hermetically sealed aluminum pans. TEM was performed on a JEOL 1200EX at $120 \mathrm{kV}$. An FEI Technai G20 TEM/STEM with a Gatan Tridium Spectrometer was used for performing energy-filtered TEM at zero loss. PXRD was performed on a Scintag XDS 2000, with a scan rate of $0.1 \%$ minute, and the full width at half maximum determined using curve fitting within Jade. UV-vis measurements were performed on a Molecular Devices Spectramax 384 Plus with quartz cuvettes. A J-KEM 210 heating controller, thermocouple, and heating mantle were used for the synthesis of the $2.6 \mathrm{~nm}$ platinum nanoparticles. Nalgene Oakridge Teflon centrifuge tubes were employed for purifying nanoparticles. Centrifugation was performed using an Eppendorf 5810. Sonication was performed in a Bandelin Sonorex Super RK 1028 B11 bath-type sonicator.

\section{Chemicals}

1,3-dibromopropane (99\%, Aldrich) and N-methyl-N,N-dioctylamine (98\%, Aldrich) were distilled from anhydrous sodium carbonate (Fisher) under high vacuum. Anhydrous methanol (99.8\%, Aldrich), anhydrous chloroform (99+\%, Aldrich) and sodium hydrosulfide hydrate (Aldrich) were used as received. The degree of hydration of the sodium hydrosulfide hydrate was determined via an NMR titration with 2, giving an approximate stoichiometry of $\mathrm{NaSH} \cdot 1.63 \mathrm{H}_{2} \mathrm{O}$ (or an approximate formula weight of 84.4 $\mathrm{g} / \mathrm{mol}$ ). Anhydrous THF was prepared by distillation from potassium (Fluka), followed by distillation from n-butyl lithium (1.6 M in cyclohexane, Aldrich) and diphenylethylene (97\%, Aldrich). Superhydride (Aldrich, 1.0 M lithium triethylborohydride in THF) and poly(ethylene glycol) 4-nonylphenyl 3-sulfopropyl ether potassium salt, 4, (Aldrich) were used as received. $\mathrm{H}_{2} \mathrm{PtCl}_{6} \cdot 6 \mathrm{H}_{2} \mathrm{O}\left(99.9 \%\right.$, Alfa Aesar), $\mathrm{HAuCl}_{4} \cdot 3 \mathrm{H}_{2} \mathrm{O}$ (ACS grade, 
Aldrich), $\mathrm{Rh}\left(\mathrm{PPh}_{3}\right)_{3} \mathrm{Cl}$ (Aldrich), palladium acetate (reagent grade, Aldrich), and 2ethylhexanoic acid ( $>99 \%$, Aldrich) were used as received. Palladium 2-ethyl hexanoate was synthesized via ligand exchange of 2-ethylhexanoic acid for the acetate of palladium acetate under high vacuum at $60^{\circ} \mathrm{C}$, using DMF (Fisher) as a solvent. The DMF was removed from the product by distillation under high vacuum.

\section{Syntheses}

N-(3-bromopropyl)-N,N-dioctyl-N-methylammonium bromide (1)

In air, 1,3-dibromopropane and N,N-dioctyl-N-methylamine were combined in a $30: 1$ molar ratio. Heating at $50{ }^{\circ} \mathrm{C}$ for 12 hours followed by vacuum distillation of the excess 1,3-dibromopropane (not exceeding $60{ }^{\circ} \mathrm{C}$ during the distillation) and followed by exposing the sample to high vacuum overnight at room temperature yielded 1 in $92 \%$ yield. The remaining $8 \%$ consisted of N,N-dioctyl-N-methylammonium bromide and allyl bromide, formed by $\beta$-hydride rearrangement of $\mathbf{1}$. The allyl bromide was removed during exposure of the sample to high vacuum. The excess 1,3-dibromopropane was recycled for subsequent syntheses.

N,N-dioctyl-N-(3-mercaptopropyl)-N-methylammonium bromide (2)

In a nitrogen glove box, $\mathbf{1}$ was dissolved in an amount of anhydrous, degassed methanol that was five times the mass of $\mathbf{1}$. Sodium hydrosulfide hydrate was added in five portions, with each addition separated by at least three hours, so that, in total, an equimolar amount had been added to the reaction. Removal of methanol in vacuum followed by dissolution of 2 in chloroform precipitated sodium bromide, which was removed by filtration. The chloroform was removed under vacuum to yield a product mixture containing the thiol $2(43 \%)$, the disulfide of $2(35 \%)$, the sulfide of $2(14 \%)$, and the side-product from the previous reaction, N,N-dioctyl-N-methyl ammonium bromide (8\%). This mixture required no further purification, as the thiol binds most strongly to platinum nanoparticles and the side products can be removed easily during nanoparticle purification.

\section{$2.0 \mathrm{~nm}$ platinum nanoparticles (3-Pt-2.0)}

$0.300 \mathrm{~g}$ of $\mathrm{H}_{2} \mathrm{PtCl}_{6} \cdot 6 \mathrm{H}_{2} \mathrm{O}$ and $0.227 \mathrm{~g}$ of $\mathrm{N}, \mathrm{N}$-dioctyl-N-(3-mercaptopropyl)-Nmethylammonium bromide, 2, were dissolved in $18 \mathrm{~g}$ of anhydrous THF in a $100 \mathrm{~mL} 1$ neck flask. After stirring for 30 minutes, $10 \mathrm{~mL}$ of $1.0 \mathrm{M}$ superhydride was injected over 10 seconds with rapid stirring. The orange-red solution turned black and bubbled furiously during the injection and warmed slightly. Stirring was continued for 30 minutes, at which point isopropanol was added to kill the excess superhydride. Solvent, triethylborane, and other volatile components were removed by rotary evaporation and exposure to high vacuum, during which vacuum typically dropped to $\sim 0.1$ mbar. The black chunky solid was transferred to a centrifuge tube and approximately $20 \mathrm{~mL}$ of methanol was added. Sonication of the centrifuge tube in a water bath for a few minutes, followed by centrifugation for 20 minutes at 7,000 rpm successfully precipitated most of the nanoparticles. The supernatant was removed and the centrifugation procedure was typically repeated once more with a 10:90 methanol:water mixture. The nanoparticles were transferred to a round bottom flask by dissolution in THF and the solution was 
rotary evaporated. Finally, vacuum was pulled on the nanoparticles until the pressure dropped to $<0.05$ mbar. This afforded a dry, black powder (see Figure S16) that was extremely soluble in a wide range of solvents.

\section{$2.7 \mathrm{~nm}$ platinum nanoparticles (3-Pt-2.7)}

$0.300 \mathrm{~g}$ of $\mathrm{H}_{2} \mathrm{PtCl}_{6} \cdot 6 \mathrm{H}_{2} \mathrm{O}$ and $0.475 \mathrm{~g}$ of 2 were dissolved in $18 \mathrm{~g}$ of anhydrous THF in a $100 \mathrm{~mL} 3$-neck flask. The flask was equipped with a reflux condenser above which nitrogen was flowed, a rubber septum, and a thermocouple. The solution was heated to $55^{\circ} \mathrm{C}$, and was maintained at that temperature for 2 hours prior to injecting the superhydride over 10 seconds. The dark red solution turned black and bubbled furiously during the injection, cooling to $51{ }^{\circ} \mathrm{C}$. Heating at $55^{\circ} \mathrm{C}$ was continued for 30 minutes, at which point the solution was cooled and isopropanol was added to kill the excess superhydride. Purification was identical to 3-Pt-2.0.

\section{$2.5 \mathrm{~nm}$ gold nanoparticles (3-Au-2.5)}

$0.470 \mathrm{~g}$ of $\mathrm{HAuCl}_{4} \cdot 3 \mathrm{H}_{2} \mathrm{O}$ and $0.575 \mathrm{~g}$ of 2 were dissolved in $42 \mathrm{~g}$ of anhydrous THF in a $250 \mathrm{~mL} 1$-neck flask. After stirring 30 minutes, $12 \mathrm{~mL}$ of superhydride was injected drop-wise over 6 minutes. The solution slowly turned from red to black during the injection. The reaction was quenched with isopropanol and purified similarly to the platinum nanoparticles. The only difference was that after the first rotary evaporation of THF and prior to the centrifugation, the nanoparticles were dissolved in chloroform, run through Whatman filter paper and subsequently a $0.2 \mu \mathrm{m}$ PTFE syringe filter, after which the chloroform was rotary evaporated. The purifications afforded a black-purple powder.

\section{$7.9 \mathrm{~nm}$ gold nanoparticles (3-Au-7.9)}

$0.199 \mathrm{~g}$ of $\mathrm{HAuCl}_{4} \cdot 3 \mathrm{H}_{2} \mathrm{O}$ and $0.425 \mathrm{~g}$ of 2 were dissolved in $18 \mathrm{~g}$ of anhydrous THF in a $100 \mathrm{~mL} 1$-neck flask. After stirring 30 minutes, $6 \mathrm{~mL}$ of superhydride was injected over 10 seconds. The solution turned from red to black during the injection and bubbled furiously. After 30 minutes, the reaction was quenched with isopropanol. Purification was identical to 3-Au-2.5. The purifications afforded a black-purple powder. This product was less stable than all other nanoparticles synthesized, and was not further characterized, except for an analysis by TEM and UV-vis spectroscopy.

\section{$1.7 \mathrm{~nm}$ palladium nanoparticles (3-Pd-1.7)}

$0.237 \mathrm{~g}$ of palladium 2-ethylhexanoate and $0.397 \mathrm{~g}$ of 2 were dissolved in $18 \mathrm{~g}$ of anhydrous THF in a $50 \mathrm{~mL}$ flask. After stirring 30 minutes, $4 \mathrm{~mL}$ of superhydride was injected over 10 seconds. The solution turned from red to black during the injection. Isopropanol was added after 5 minutes to kill the excess superhydride. The purification was identical to 3-Pt-2.0, except that the nanoparticles were centrifuged first in a 90:10 methanol: $\mathrm{H}_{2} \mathrm{O}$ solution, followed by three centrifugations in a 10:90 methanol: $\mathrm{H}_{2} \mathrm{O}$ solution. This yielded a black powder.

\section{$2.0 \mathrm{~nm}$ rhodium nanoparticles (3-Rh-2.0)}

$0.401 \mathrm{~g}$ of $\mathrm{Rh}\left(\mathrm{PPh}_{3}\right)_{3} \mathrm{Cl}$ and $0.405 \mathrm{~g}$ of 2 were dissolved in $35 \mathrm{~g}$ of toluene and $5 \mathrm{~g}$ of THF. After stirring for 1 hour, $4 \mathrm{~mL}$ of superhydride was injected over 10 seconds. The solution bubbled some at this point but the reddish-black solution did not change 
color noticeably. After stirring 10 minutes, isopropanol was added. Purification was identical to 3-Pd-1.7. We found it difficult to remove the $\mathrm{PPh}_{3}$ from the nanoparticles via this purification procedure. Accordingly, the TGA results show a higher organic content as compared with other nanoparticles.

\section{Liquid nanoparticles (5)}

The synthesis of 5 for all nanoparticles was identical, irregardless of the size or composition of $\mathbf{3}$. To determine the ratio of $\mathbf{3}$ and $\mathbf{4}$ to mix together, TGA was performed on 3 under flowing $\mathrm{N}_{2}$. The mass loss was attributed to the thiol 2 bound to the surface of the metal nanoparticles. Based on this, $\mathbf{4}$ was added to 3 to provide a 1:1 molar ratio between $\mathbf{4}$ and $\mathbf{2}$. Chloroform was added to dissolve the reagents and the solution was stirred for several hours. To remove the $\mathrm{KBr}$ salt, water was then added to the stirring solution, and the reaction was stirred for several more hours. The water was removed by pipet, and more water was added to the flask. After stirring a few minutes, the water was again removed. This was then repeated once more. The solution was then rotary evaporated and then exposed to high vacuum until the pressure reached $<0.05$ mbar. All nanoparticles thus synthesized were viscous, homogeneous liquids at room temperature.

Analysis of the products 5 by ${ }^{1} \mathrm{H}$ NMR, as in Fig. S10, reveals that there is almost exactly a 1:1 correspondence between the thiol cation and the sulfonate anion. We calculated this ratio by summing the integrations of the thiol peaks $\mathrm{d}$, e, and 1 and summing the integrations of the sulfonate peaks $\mathrm{v}, \mathrm{u}, \mathrm{s}, \mathrm{m}, \mathrm{o}, \mathrm{x}, \mathrm{n}$, and $\mathrm{F}$. Division of these sums by the expected number of hydrogens for these peaks gives a thiol:sulfonate ratio of 1:0.989. Because the purification of the nanoparticles involved multiple, extensive washings with water which would remove unreacted $\mathbf{4}$, we conclude that nearly all bromide anion (98.9\%) is replaced with sulfonate anion.

\section{NMR Spectra}

The following NMR spectra and corresponding molecules (Figures S1-S11) show ${ }^{1} \mathrm{H}$ and

${ }^{13} \mathrm{C}$ NMR spectra for 1, 2, 3-Pt-2.0, and 5-Pt-2.0. 


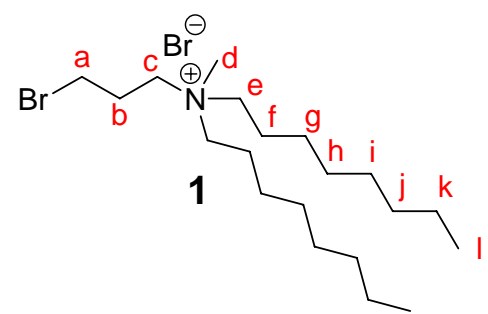

Figure S1. Key of NMR labels in Figures S2 and S3 for N-(3-bromopropyl)-N,Ndioctyl-N-methylammonium bromide (1).

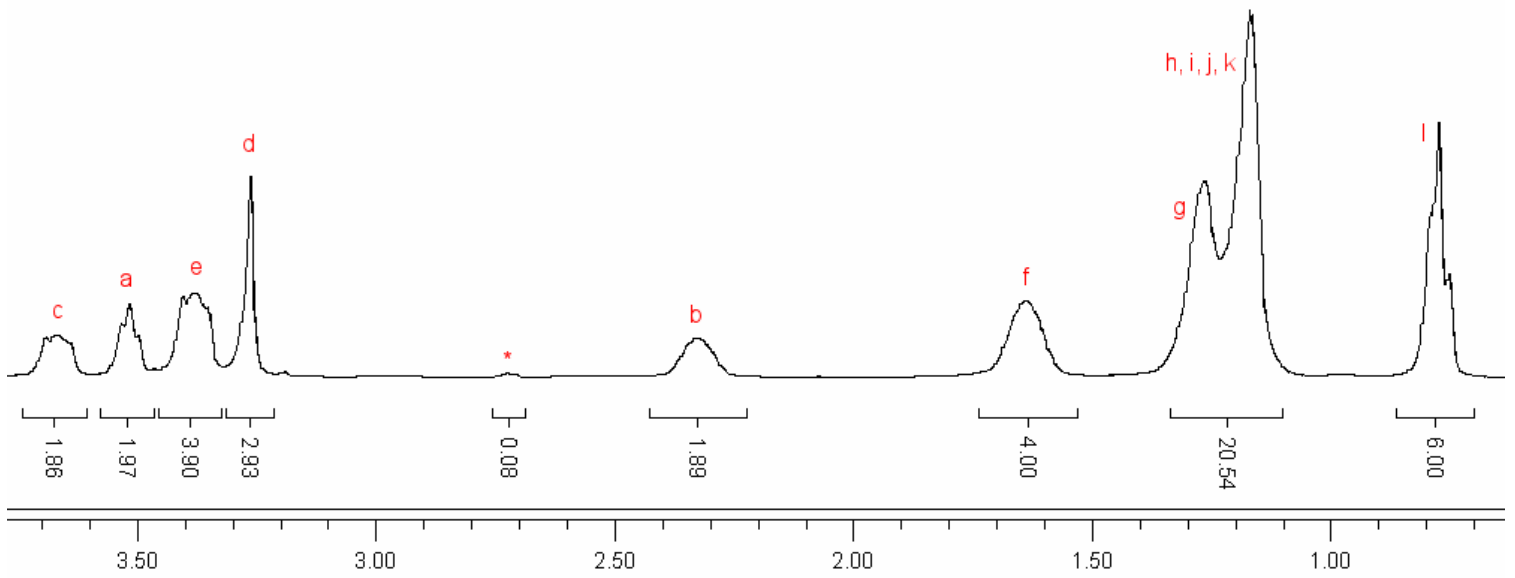

Figure S2. ${ }^{1} \mathrm{H}$ NMR spectrum of N-(3-bromopropyl)-N,N-dioctyl-N-methylammonium bromide (1). The * denotes the $8 \%$ side product N,N-dioctyl-N-methylammonium bromide.
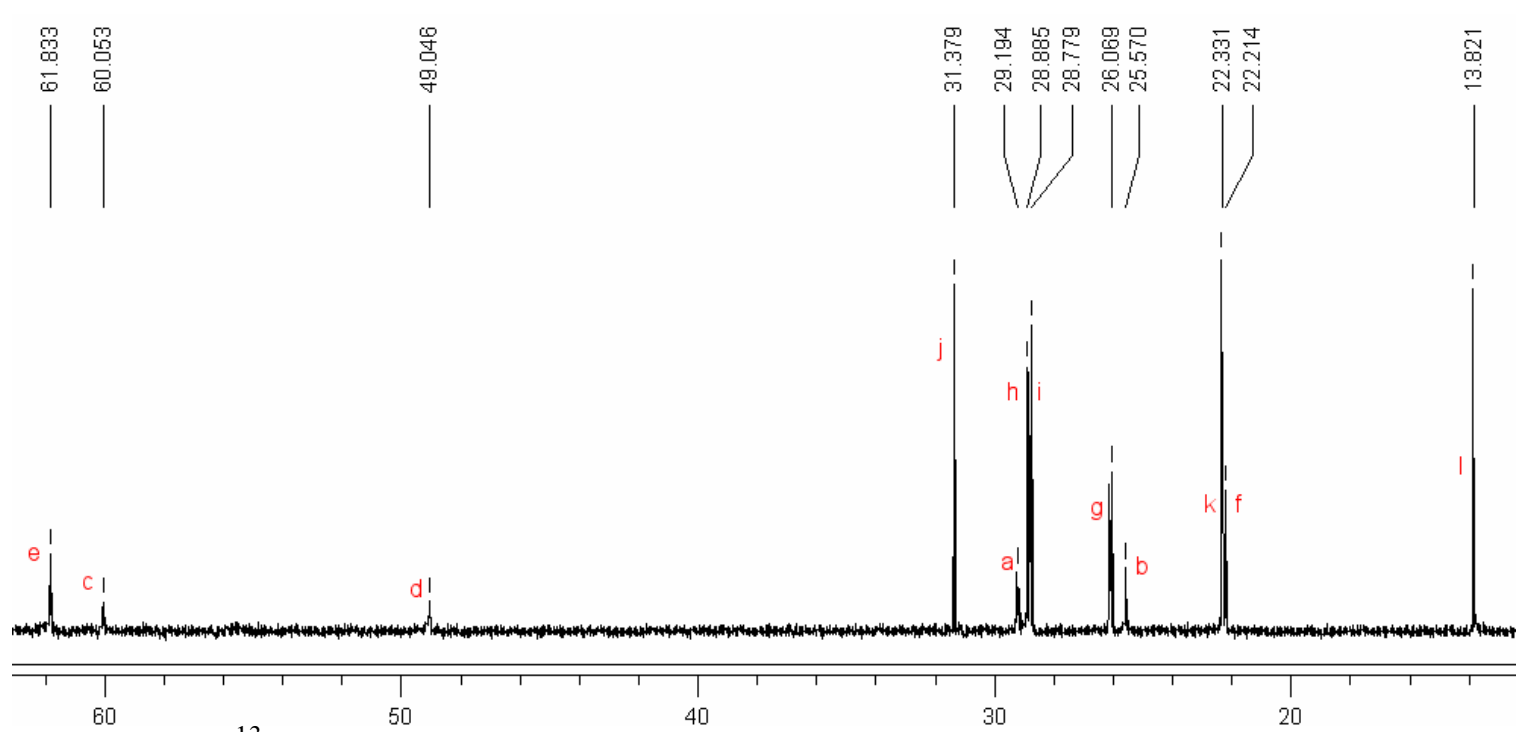

Figure S3. ${ }^{13} \mathrm{C}$ NMR spectrum of N-(3-bromopropyl)-N,N-dioctyl-Nmethylammonium bromide (1). 

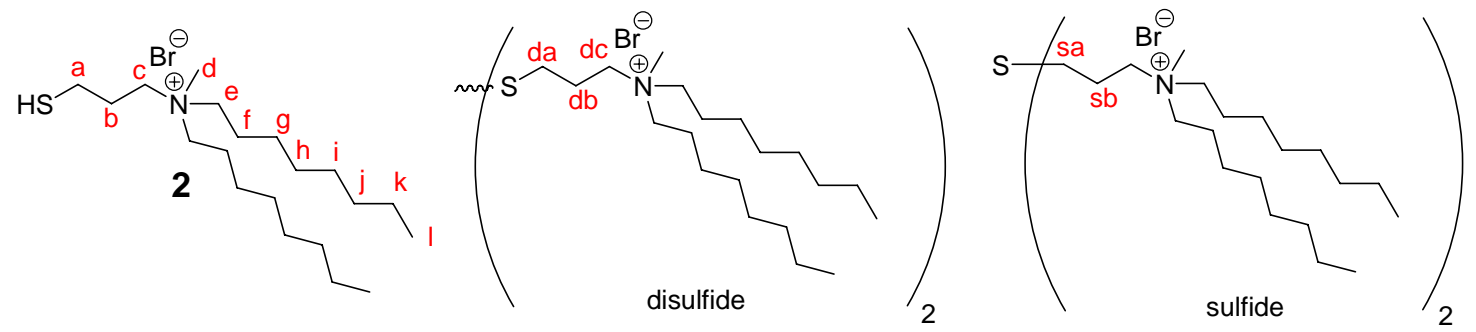

Figure S4. Key of NMR labels in Figures S5-S8 for N,N-dioctyl-N-(3-

mercaptopropyl)-N-methylammonium bromide (2) and the corresponding disulfide and sulfide. In Figures S5 and S6, the disulfide and sulfide have prefixes $d$ and $s$, respectively.

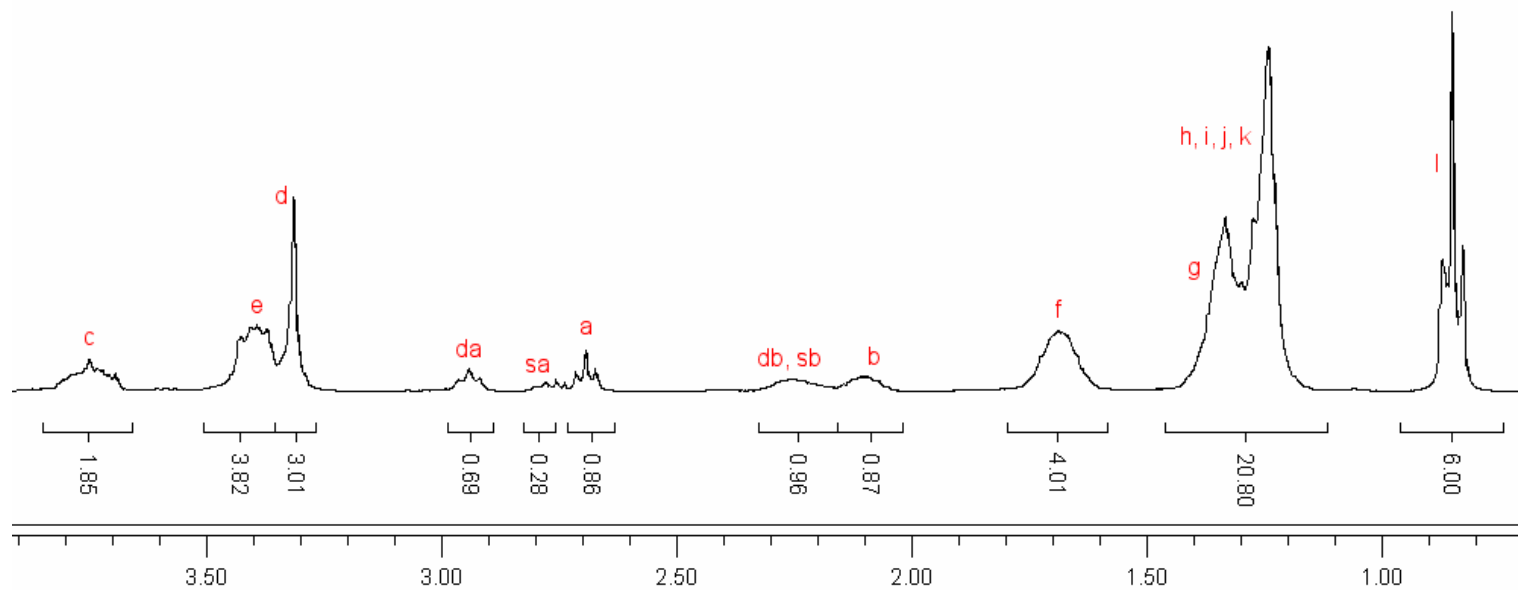

Figure S5. ${ }^{1} \mathrm{H}$ NMR spectrum of N,N-dioctyl-N-(3-mercaptopropyl)-N-

methylammonium bromide (2). The yield was calculated by integrating the "a" peaks and dividing by 2 . Accordingly, the thiol yield was $43 \%$, the disulfide yield was $35 \%$, and the sulfide yield was $14 \%$. The remaining product is the $8 \%$ side product N,Ndioctyl-N-methylammonium bromide, which appears as a small peak between sa and a.
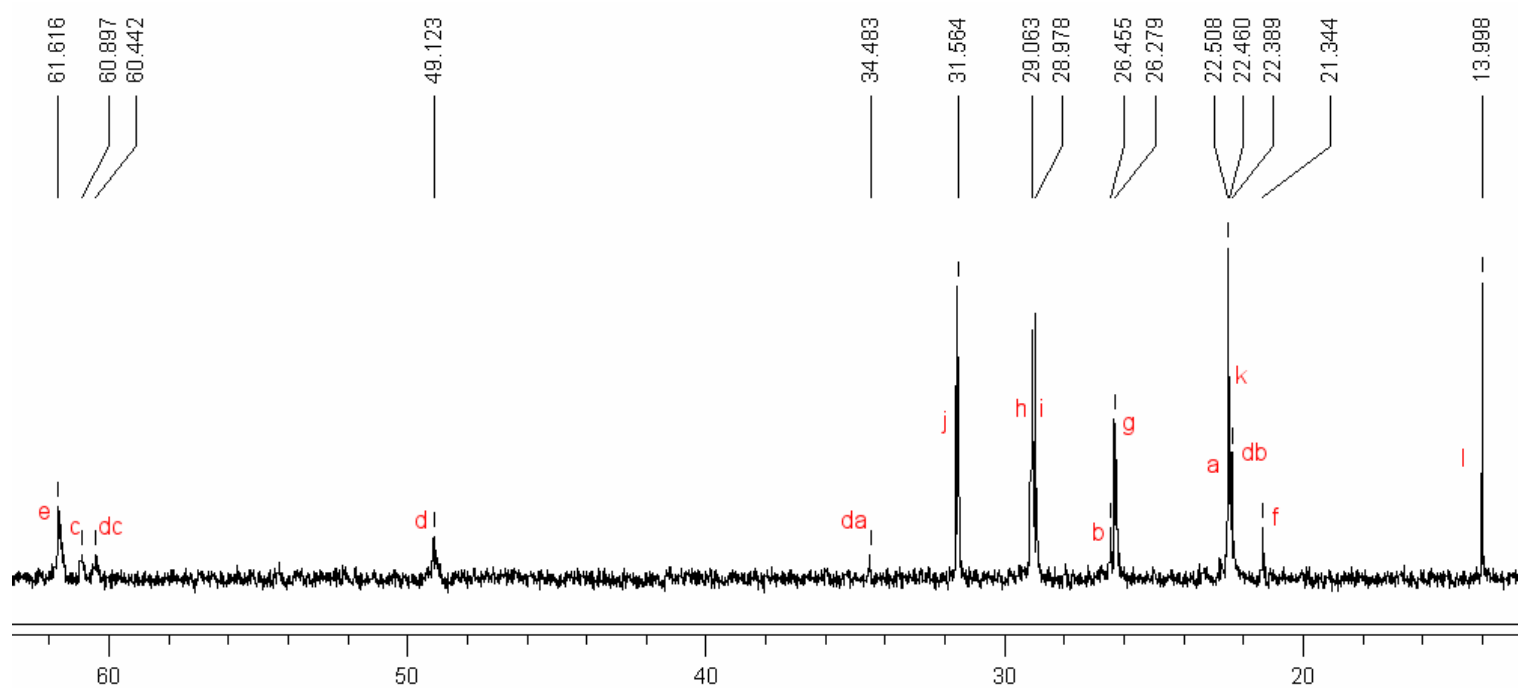

Figure S6. ${ }^{13} \mathrm{C}$ NMR spectrum of N,N-dioctyl-N-(3-mercaptopropyl)-Nmethylammonium bromide (2). 


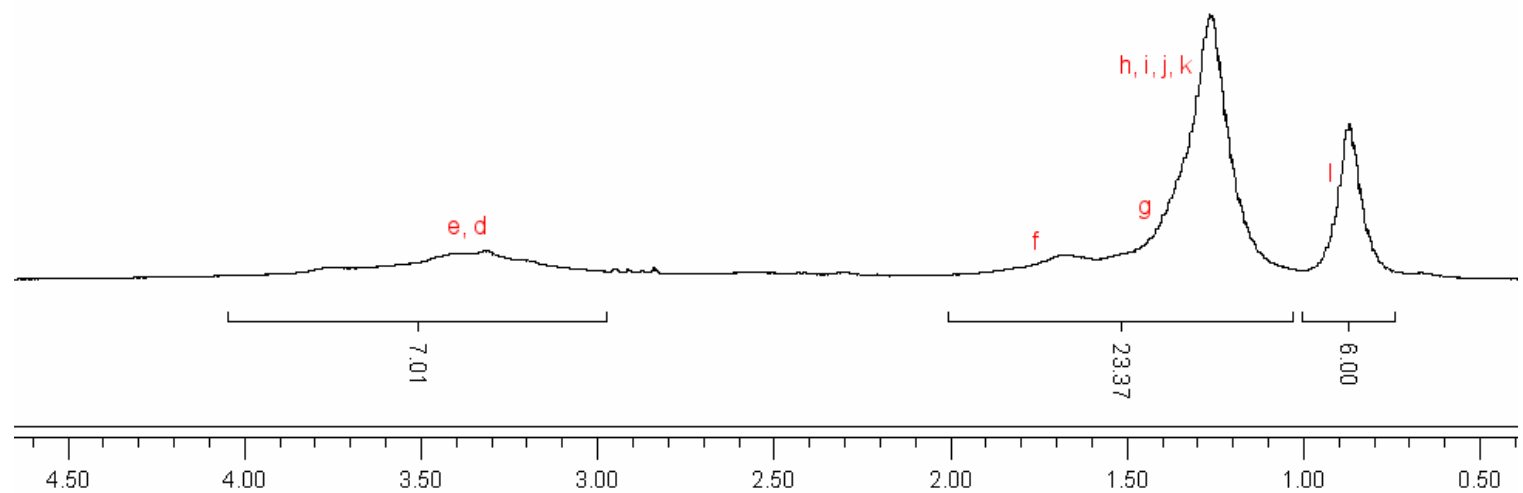

Figure S7. ${ }^{1} \mathrm{H}$ NMR spectrum of 3-Pt-2.0. Note that peaks a, b, and c do not appear, which is due to their extremely rapid relaxation. The remaining peaks decrease in width as the corresponding protons are farther removed from the nanoparticle surface. At this stage, the disulfide, sulfide, and side product N,N-dioctyl-N-methylammonium bromide have been removed.

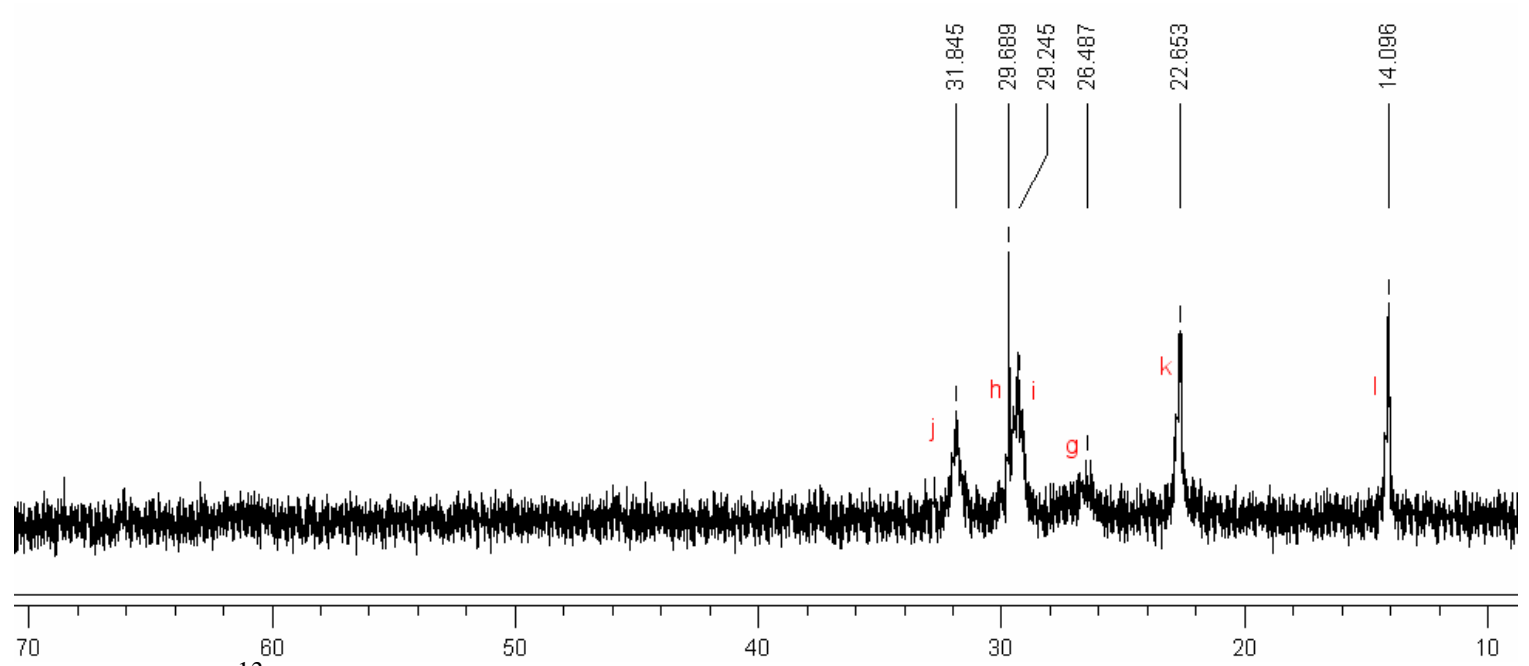

Figure S8. ${ }^{13} \mathrm{C}$ NMR spectrum of 3-Pt-2.0. Note that peaks a through $\mathrm{f}$ do not appear, owing to their proximity to the nanoparticle surface. The restricted environment at the nanoparticle surface, due to the dense packing of thiols and the sterically-restrictive architecture of the ligand, causes spin-spin relaxations to occur more rapidly for the protons nearest to the surface. 


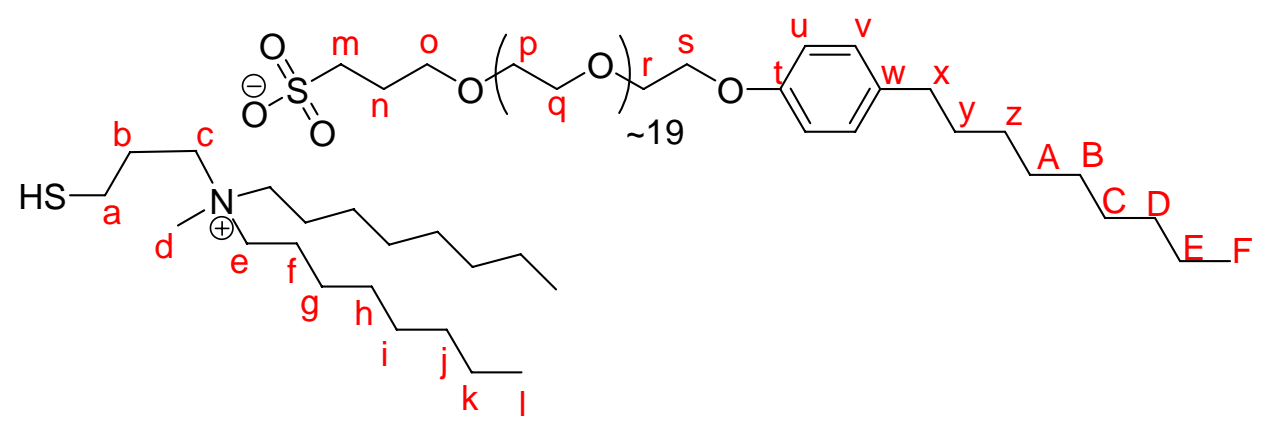

Figure S9. Key of NMR labels in Figures S10 and S11 for N,N-dioctyl-N-(3mercaptopropyl)-N-methylammonium poly(ethylene glycol) 4-nonylphenyl 3-sulfopropyl ether, which is the ligand on (5).

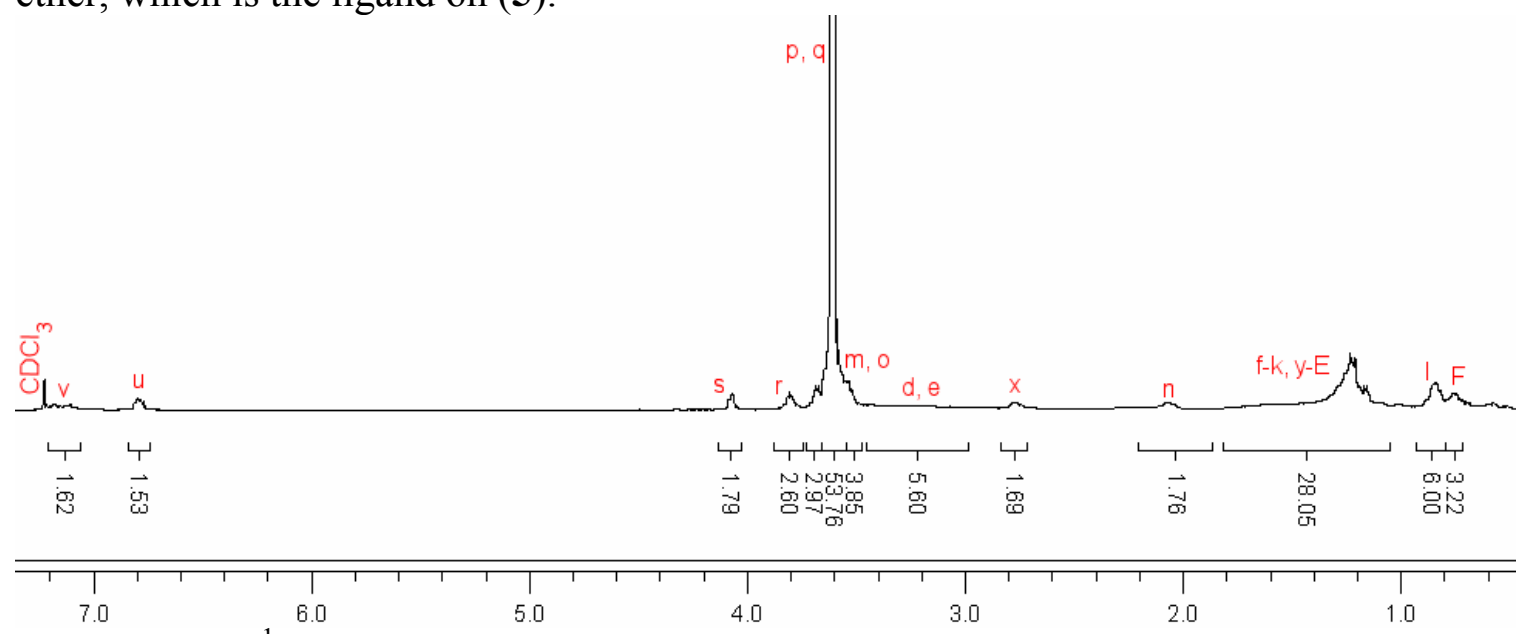

Figure S10. ${ }^{1} \mathrm{H}$ NMR spectrum of 5-Pt-2.0.

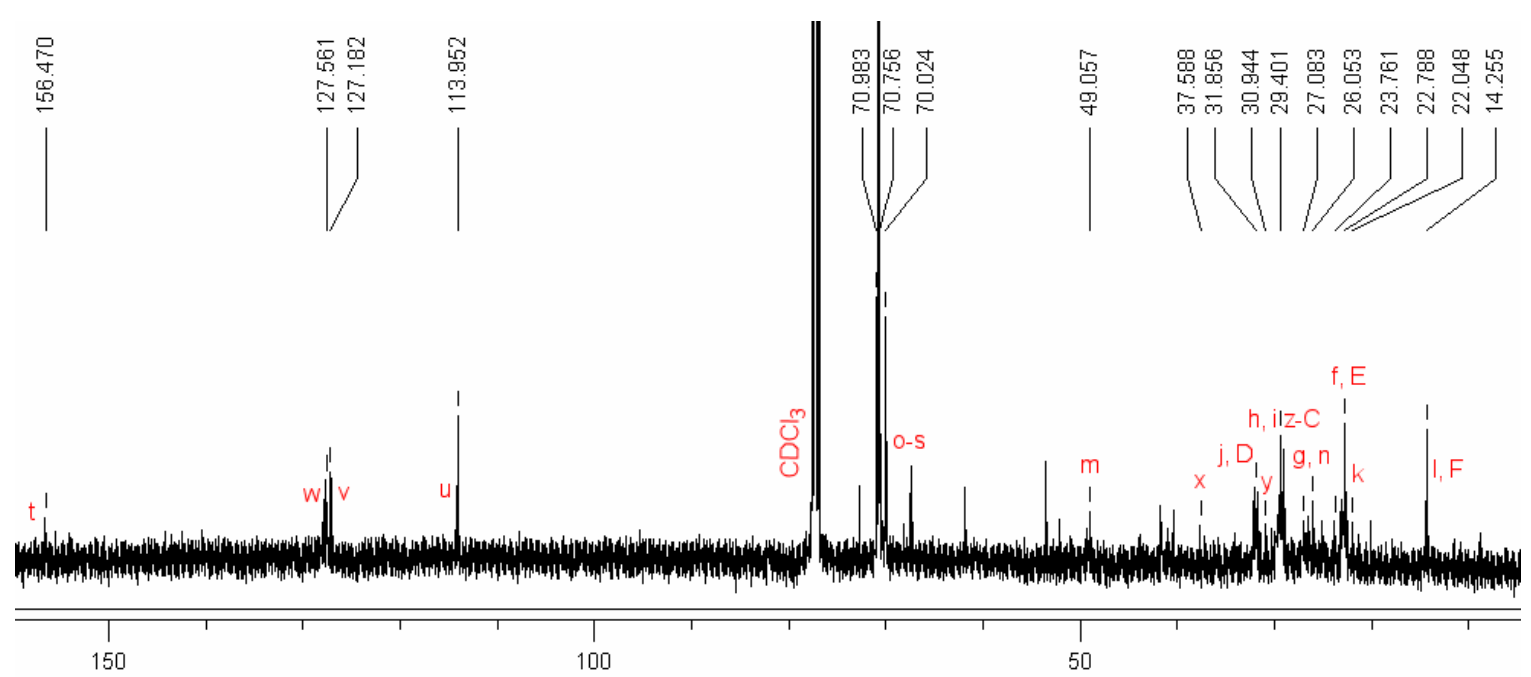

Figure S11. ${ }^{13} \mathrm{C}$ NMR spectrum of 5-Pt-2.0. 


\section{TEM Images and Size Distributions}

TEM images of the samples not shown in the communication are included below (Figure S12). Additionally, histograms for all nanoparticles are displayed here.
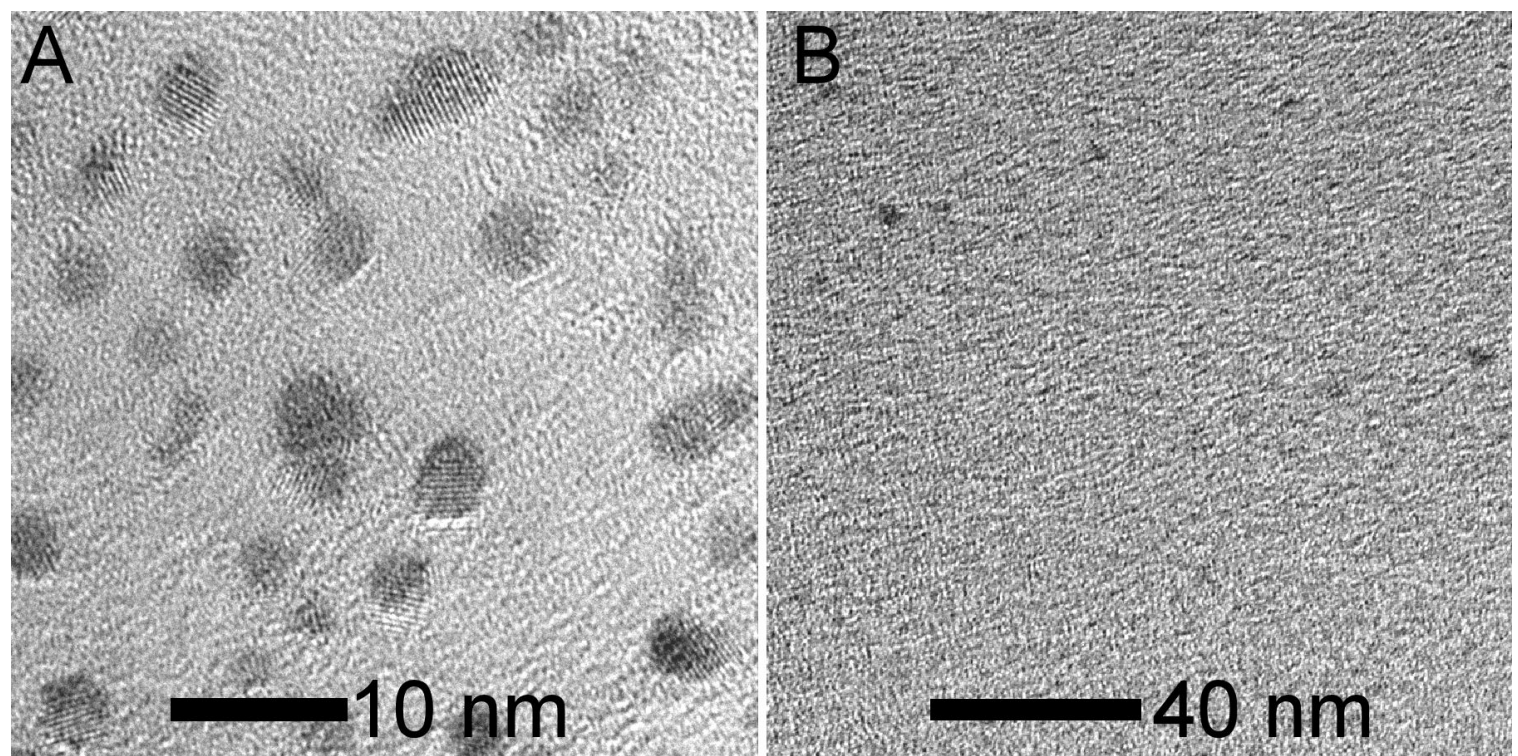

Figure S12. TEM images of (A) $2.7 \mathrm{~nm}$ platinum nanoparticles (3-Pt-2.7) and (B) 2.0 nm rhodium nanoparticles (3-Rh-2.0). 


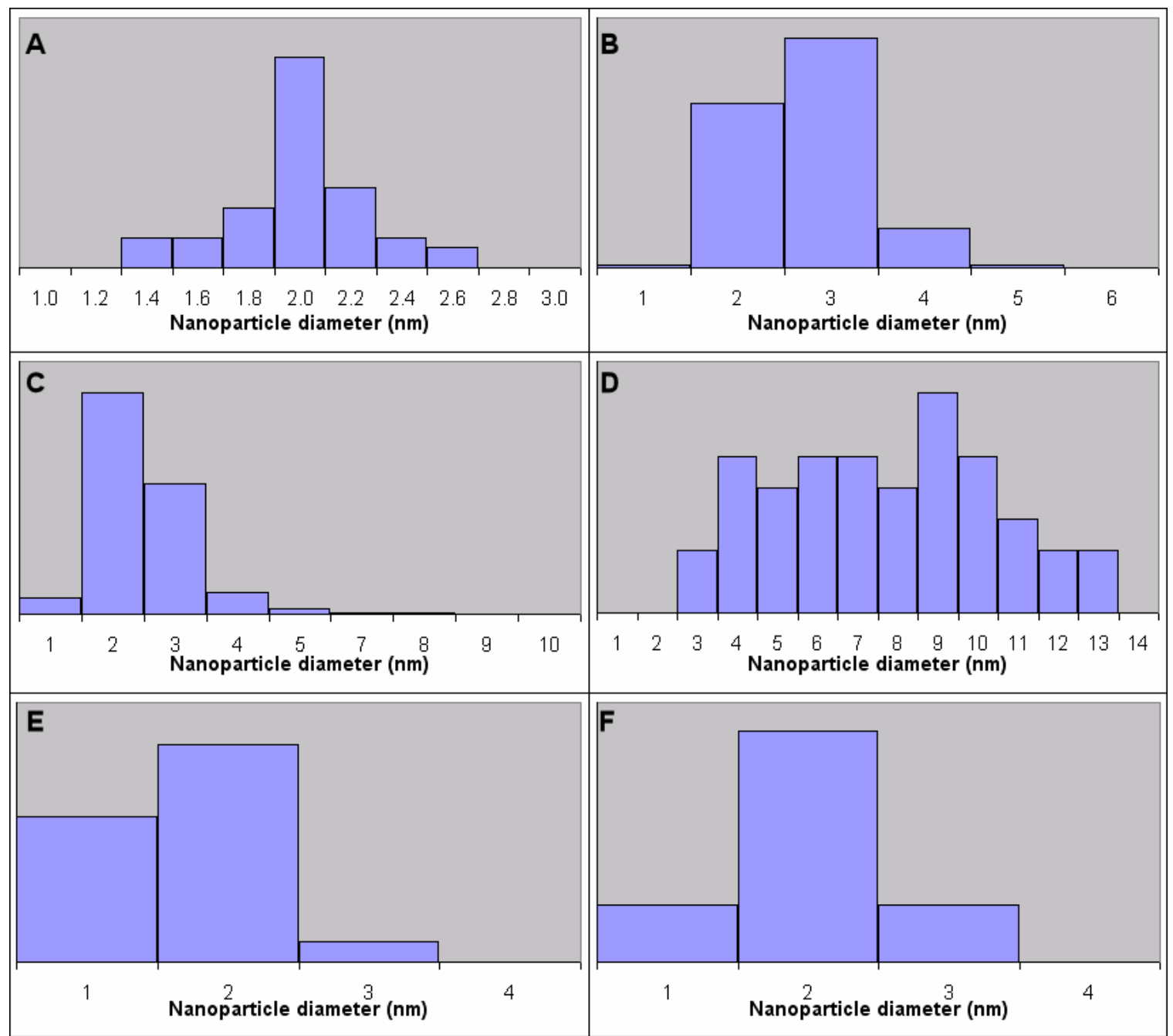

Figure S13. Size distributions of (A) $2.0 \pm 0.3 \mathrm{~nm}$ platinum nanoparticles (3-Pt-2.0), (B) $2.7 \pm 0.6 \mathrm{~nm}$ platinum nanoparticles (3-Pt-2.7), (C) $2.5 \pm 0.8 \mathrm{~nm}$ gold nanoparticles (3-Au-2.5), (D) $7.9 \pm 3.0 \mathrm{~nm}$ gold nanoparticles (3-Au-7.9), (E) $1.7 \pm 0.4 \mathrm{~nm}$ palladium nanoparticles (3-Pd-1.7), and (F) $2.0 \pm 0.4 \mathrm{~nm}$ rhodium nanoparticles (3-Rh-1.7). 
Figure S14 shows the TGA traces of 3-Pt-2.0 and 5-Pt-2.0. In general, mass loss depended on metal density (mass loss of Pd $>\mathrm{Pt}$ ), nanoparticle size (mass loss of 3-Pt2.0 $>$ 3-Pt-2.7), counter ion (mass loss of sulfonate $>$ bromide), and sample purity (mass loss of 3-Rh-2.0 > 3-Pd-1.7). Table 1 compiles all samples analyzed. Interestingly, table 1 shows that by swapping the bromide counter ion for the sulfonate counter ion, the temperature stability is enhanced to some degree. The low decomposition temperature of these nanoparticles is likely due to the catalytic role that late transition metals typically play in the combustion of hydrocarbons.

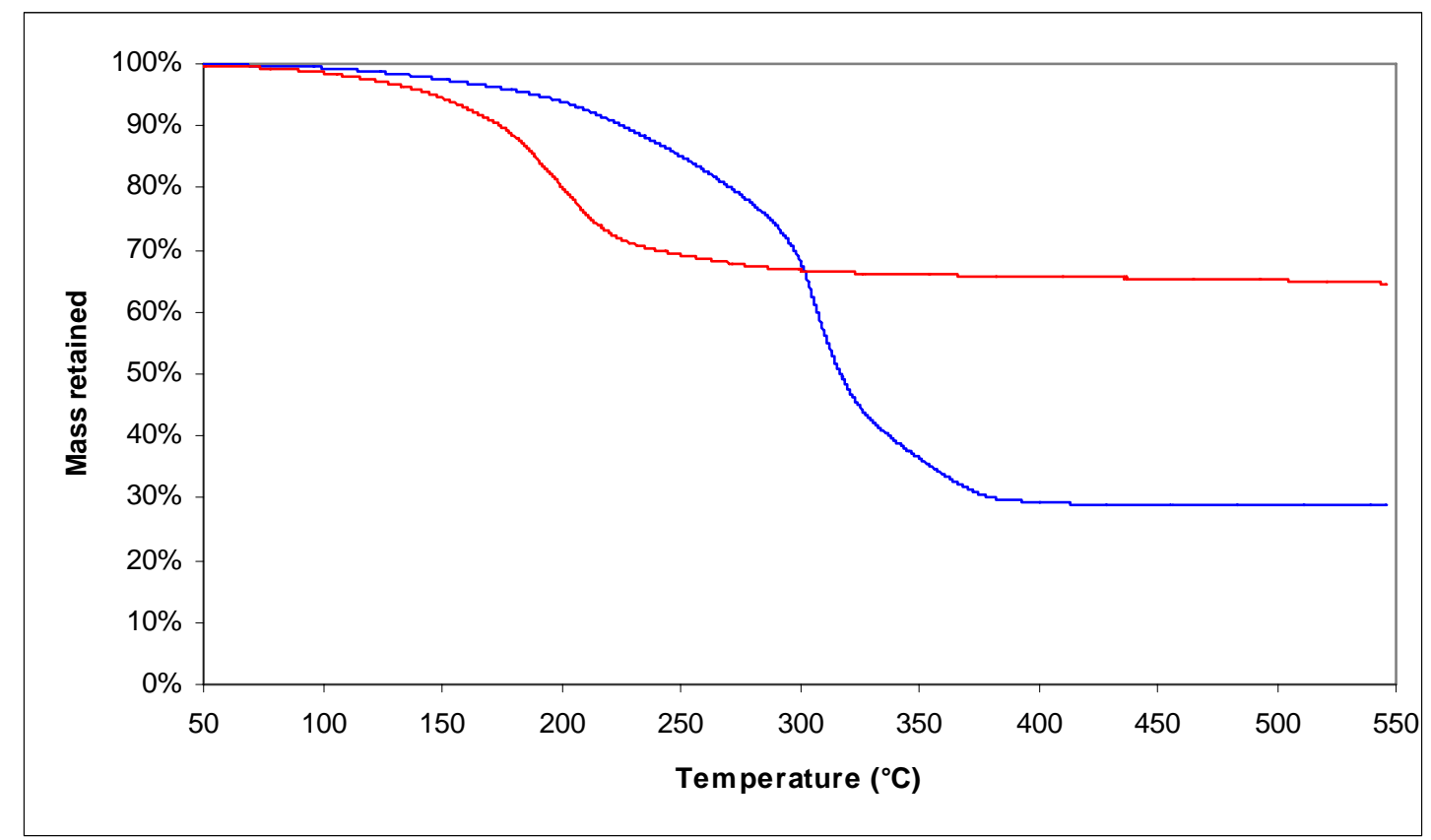

Figure S14. Red trace shows 3-Pt-2.0, with a total mass retained of $64 \%$, and blue trace shows 5-Pt-2.0, with a total mass retained of $29 \%$.

Table S1. TGA results of all nanoparticles studied.

\begin{tabular}{|c|c|c|}
\hline Sample & Mass retained & Onset of decomposition $\left({ }^{\circ} \mathbf{C}\right)^{\dagger}$ \\
$3-\mathrm{Pt}-2.0$ & $64 \%$ & 142 \\
$5-\mathrm{Pt}-2.0$ & $29 \%$ & 159 \\
$3-\mathrm{Pt}-2.7$ & $69 \%$ & 162 \\
$5-\mathrm{Pt}-2.7$ & $36 \%$ & 184 \\
$3-\mathrm{Au}-2.5$ & $67 \%$ & 193 \\
$5-\mathrm{Au}-2.5$ & $25 \%$ & 220 \\
$3-\mathrm{Pd}-1.7$ & $53 \%$ & 135 \\
$5-\mathrm{Pd}-1.7$ & $22 \%$ & 170 \\
$3-\mathrm{Rh}-2.0$ & $39 \%$ & 120 \\
$5-\mathrm{Rh}-2.0$ & $12 \%$ & 151 \\
\hline
\end{tabular}

Onset of decomposition is defined here as the temperature at which $5 \%$ of mass has been lost. 


\section{DSC}

All samples were analyzed with a heat/cool/heat protocol in which the upper and lower temperatures were $-150^{\circ} \mathrm{C}$ and $130^{\circ} \mathrm{C}$, respectively. The scan rate was $10^{\circ} \mathrm{C} /$ minute. No melting behavior was observed for the samples with a bromide counter ion.

The crystallization and melting kinetics of the ligand are sped by the presence of the nanoparticles. However, samples 4, 5-Pt-2.7, and 5-Au-2.5, (the samples with the largest nanoparticles) undergo crystallization primarily on the heating cycle, whereas the others undergo crystallization primarily on the cooling cycle.

Because the liquid-to-solid transition is often split between heating and cooling cycles, as in the case of 4, 5-Pt-2.7, and 5-Pd-1.7, the measured enthalpy is lower than the solid-to-liquid transition. The magnitude of the enthalpy is closely related to the weight fraction of sulfonate ligand $\mathbf{4}$ added. The only exception to this is 5-Rh-2.0, where the latent $\mathrm{PPh}_{3}$ may suppress crystallization.

Table S2. DSC results of all liquid-type nanoparticles.

\begin{tabular}{|c|c|c|c|c|}
\hline $\begin{array}{c}\text { Sample } \\
\text { Sulfonate } 4\end{array}$ & $\begin{array}{c}\mathbf{T}_{\mathbf{m}}(\mathbf{l} \rightarrow \mathbf{s})\left({ }^{\circ} \mathbf{C}\right) \\
-21\end{array}$ & $\begin{array}{c}\mathbf{T}_{\mathrm{m}}(\mathrm{s} \rightarrow \mathbf{l})\left({ }^{\circ} \mathbf{C}\right) \\
29\end{array}$ & $\begin{array}{c}\Delta \mathbf{H}(\mathbf{l} \rightarrow \mathbf{s})(\mathrm{J} / \mathrm{g}) \\
44\end{array}$ & $\underset{63}{\Delta \mathbf{H}(\mathrm{s} \rightarrow \mathrm{l})(\mathrm{J} / \mathrm{g})}$ \\
\hline 5-Pt-2.0 & -11 & 16 & 32 & 34 \\
\hline 5-Pt-2.7 & -17 & 26 & 13 & 27 \\
\hline 5-Au-2.5 & -35 & 17 & 28 & 31 \\
\hline $5-\mathrm{Pd}-1.7$ & -12 & 21 & 32 & 42 \\
\hline 5-Rh-2.0 & -3 & 17 & 10 & 8 \\
\hline
\end{tabular}

Photographs of samples

The following Figures S15-S17 show photographs of the nanoparticles.

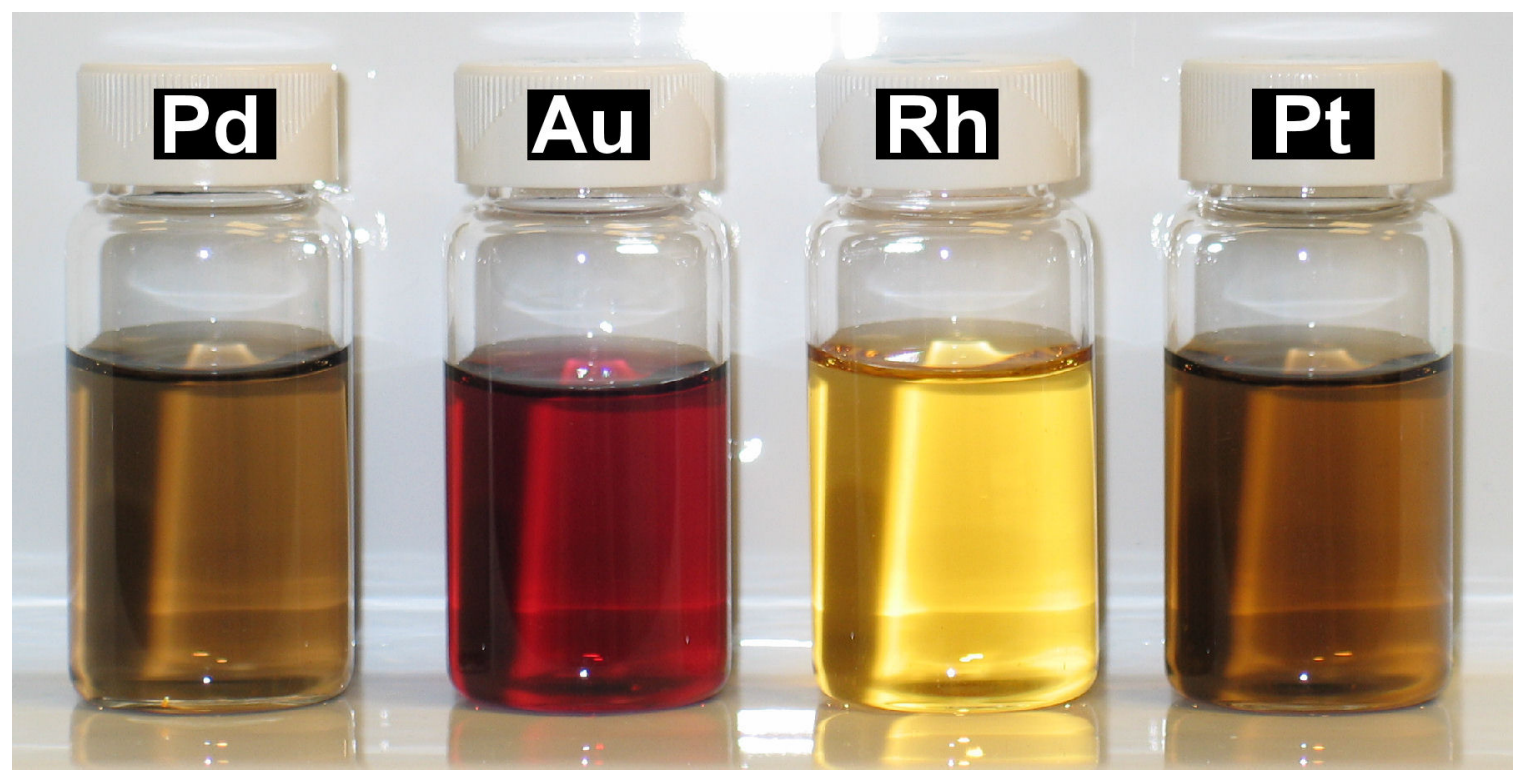

Figure S15. Photograph of 3 dissolved in THF, with the camera flash turned on. 


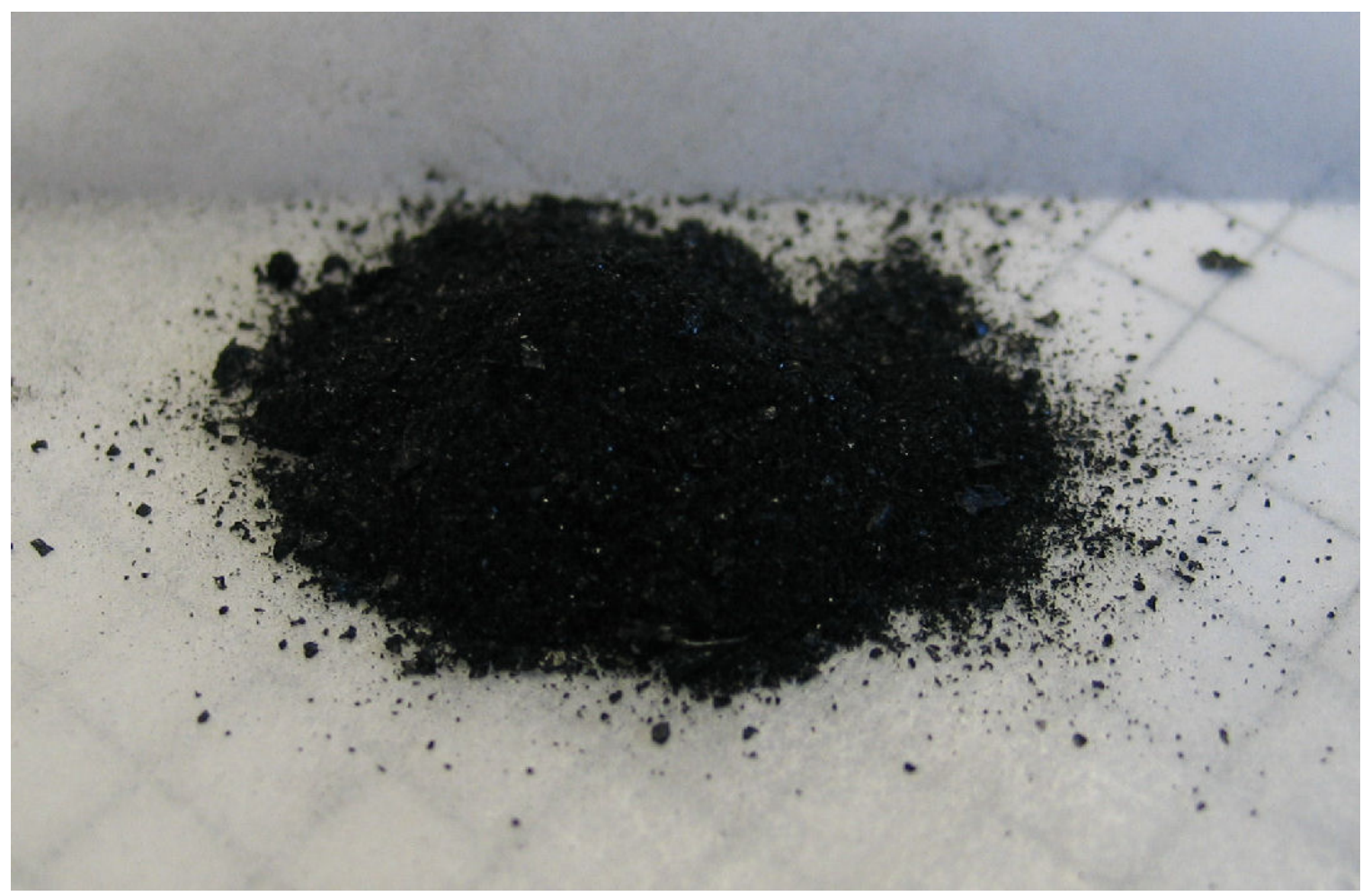

Figure S16. Photograph of $0.5 \mathrm{~g}$ of 3-Pt-2.0.

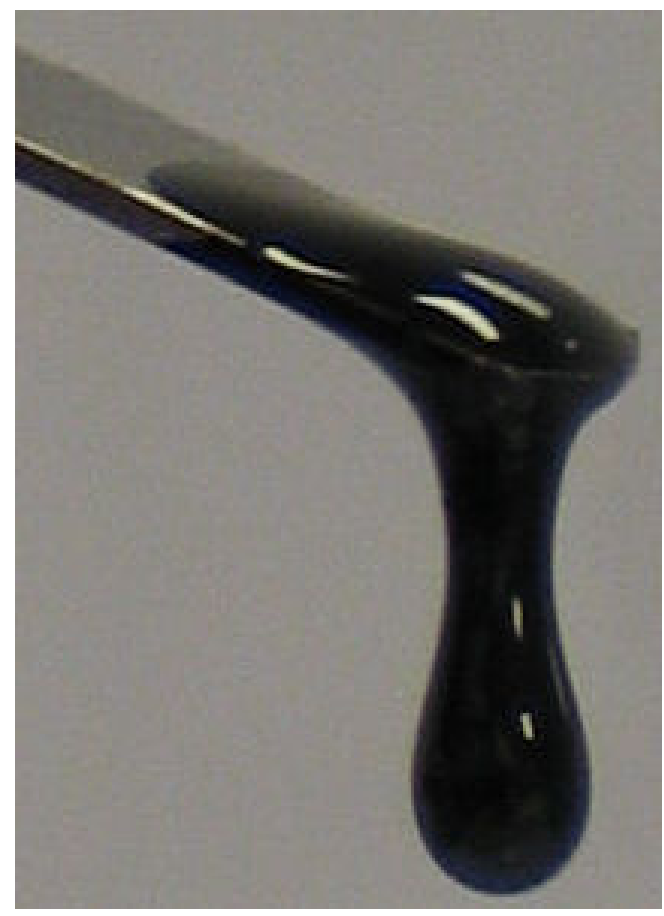

Figure S17. Photograph of a drop of 5-Pt-2.0 at the tip of a spatula, taken at room temperature. All nanoparticles $\mathbf{5}$ flowed as a fluid at room temperature. 